\title{
High efficiency, Diode Pumped 170 W Nd:YAG ceramic slab laser
}

\author{
A. Lapucci \\ antonio.lapucci@ino.it
}

M. Ciofini

M. Pucci

M. D’Uva

CNR - National Research Council, Istituto Nazionale di Ottica,Largo Enrico Fermi 6, I-50125 Firenze, Italy

CNR - National Research Council, Istituto Nazionale di Ottica,Largo Enrico Fermi 6, I-50125 Firenze, Italy

CNR - National Research Council, Istituto Nazionale di Ottica,Largo Enrico Fermi 6, I-50125 Firenze, Italy

CNR - National Research Council, Istituto Nazionale di Ottica,Largo Enrico Fermi 6, I-50125 Firenze, Italy

A new thin-slab ceramic Nd:YAG laser is presented based on a non-conventional zig-zag configuration. The slab is shaped in such a way that the beam propagates internally bouncing on the thin lateral faces (Edge zig-zag). Preliminary experiments have demonstrated a power extraction of $170 \mathrm{~W}(\mathrm{CW})$ at an optical conversion efficiency of $36 \%$. Measurements have also been performed to assess the propagation properties, at least in the thinner transverse direction, of the beam extracted with a simple stable resonator. This laser format appears as a good competitor of the more traditional face-pumped or edge-pumped zig-zag slab laser schemes. [D0I: http://dx.doi.org/10.2971/jeos. 2011.11047]

Keywords: Diode Pumped Solid State Lasers, Slab Lasers, Zig-Zag Slab Lasers

\section{INTRODUCTION}

High-power high-efficiency solid state lasers are always limited by the onset of deleterious effects such as thermal lensing, stress birefringence or biaxial focusing. From the early 1970s zig- zag slab designs have been suggested to reduce thermally induced effects [1]. In this design a laser beam is propagated through a slab of active material at an angle with respect to the slab axis and bounces inside the slab thanks to total internal reflections (TIRs). Pumping and cooling can be parallel and uniaxial, through the large lateral slab faces, in principle drastically reducing the effect of thermal distortions. From the 1990s several diode-pumped devices have been constructed using this scheme [2]-[4]. Recently high energy multi Joule versions for Inertial Fusion Applications, like the HALNA Laser [5], or high power multi Kilowatt ones, like the ThinZag Laser [6], have been developed based on a face-pumped zig-zag slab geometry. It is indeed clear that the slab design is perfectly suited for the scaling up of the extracted power by simply increasing the width of the slab rectangular cross-section. High brightness sources can be obtained using Hybrid Stable-Unstable Resonators (HSURs) [7]-[9].

The main draw-back of this face-pumped zig-zag slab laser is the limited laser efficiency it usually allows [10]-[11] especially when compared to end-pumped solutions [12]-[13]. Moreover the parallel direction of pumping and cooling (see scheme A in Fig. 1) is obtained by letting the pump radiation pass through the cooling fluid. This fact determines a higher complexity of the cooling interface management, often limits the pumping simplicity and efficiency, and eventually leads to a limited thermo-mechanical stability [14].

In 2001 R.L. Byer and his group [15]-[16] proposed a different solution called edge-pumping (Fig. 1 scheme B) in which the rectangular slab is still uniformly cooled on the large TIR faces, but the pump power is introduced from the narrow non-TIR faces along the slab width, transverse to the direction of the optical propagation and to the thermal gradient. This geometry produces relevant power extraction efficiencies but results less effective than the face pumped scheme in averaging inhomogeneities. Indeed in the edge pumped format zig-zag propagation smears out refractive index variations due to thermal gradients, but does not act on the non uniform gain distribution.

In this work we report on the performance of a new version of thin zig-zag laser, whose principle is depicted in the $C$ scheme of Fig. 1. In this laser the Brewster angle faces determine a zig-zag path in the slab producing bounces on the thin lateral faces where, like in the case of edge-pumped slabs, the pump radiation input takes place. Cooling is obtained by contacting cold plates to the larger lateral faces. In this way the zig-zag propagation acts to average inhomogeneities due to the exponential absorption profile at the expense of not averaging the parabolic gradient in the cooled direction. In fact these 


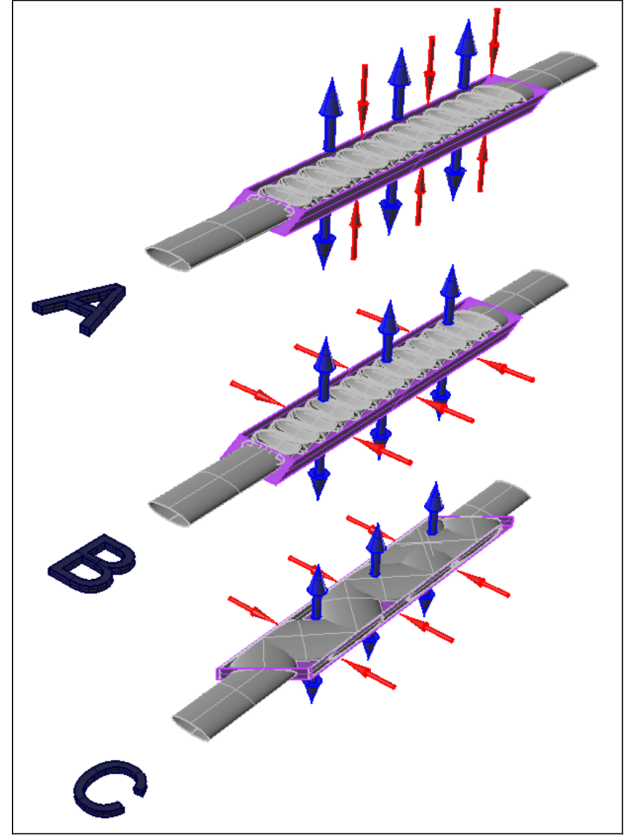

FIG. 1 Schematic drawing of different zig-zag slab laser configurations: A) Face Pumped, B) Edge Pumped and C) Edge Zig-zagged. Thick arrows (blue) indicate the cooling direction, thin arrows (red) indicate the pumping direction.

gradients produce a cylindrical thermal lens effect that can be compensated in the optical resonator scheme. A further advantage of this zig-zag propagation is that it allows the intra-cavity beam to better overlap with the highly pumped edges of the slab. This fact eventually enhances the extraction efficiency. A similar geometry was adopted in the 1990s for a low power miniature slab laser called the Coplanar-Pumped Folded Slab (CPFS) [17], [18]. To the best of our knowledge, there has never been an attempt to scale it up to high power extractions, so far.

For shortness reasons, and to distinguish it from the traditional face-pumped (A in Fig. 1) and edge-pumped (B in Fig. 1) schemes, we will call this one, the "Edge zig-zagged" scheme.

\section{The edge zig-zagged laser: preliminary experimental results}

Our active medium consists in a $2 \mathrm{~mm}$ thick, $10 \mathrm{~mm}$ wide Nd:YAG (1\% at. doping) ceramic slab (Produced by Konoshima Chemicals - Baikowski Group) with a parallelogram foot-print and a center-line length of $67 \mathrm{~mm}$. A picture of the thin slab with optical path traced by an He-Ne aiming beam is shown in Fig. 2. Polycrystalline YAG has shown higher thermo-mechanical properties and a higher homogeneity when compared to single crystals. Thus the use of a polycrystalline medium enhances the maximum thermal loading the slab can withstand [19], [20].

The Brewster angle oriented end faces produce a zig-zag propagation inside the slab with two bounces on each thin lateral face $(2 \mathrm{~mm} \times 67 \mathrm{~mm})$. These same two faces are

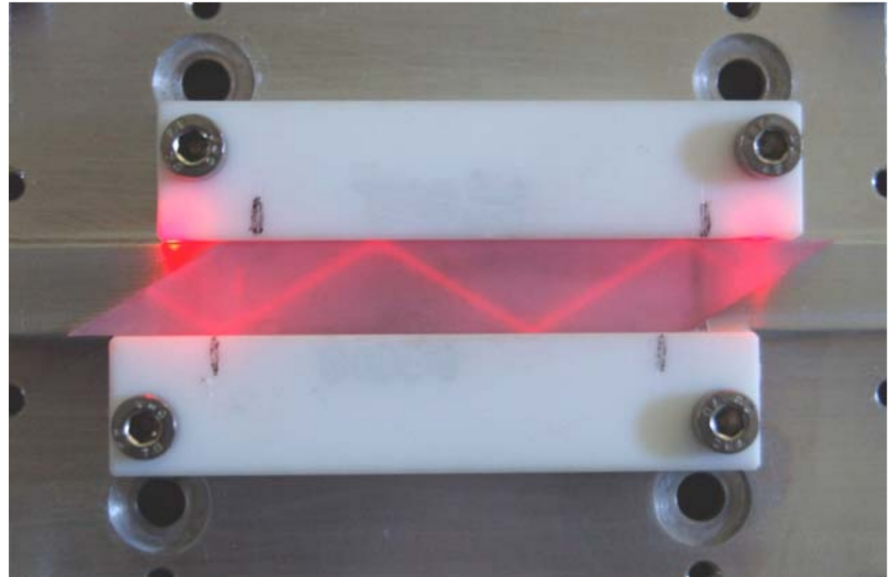

FIG. 2 Portrait of the edge zig-zagged slab with internal path traced by an He-Ne aiming beam.

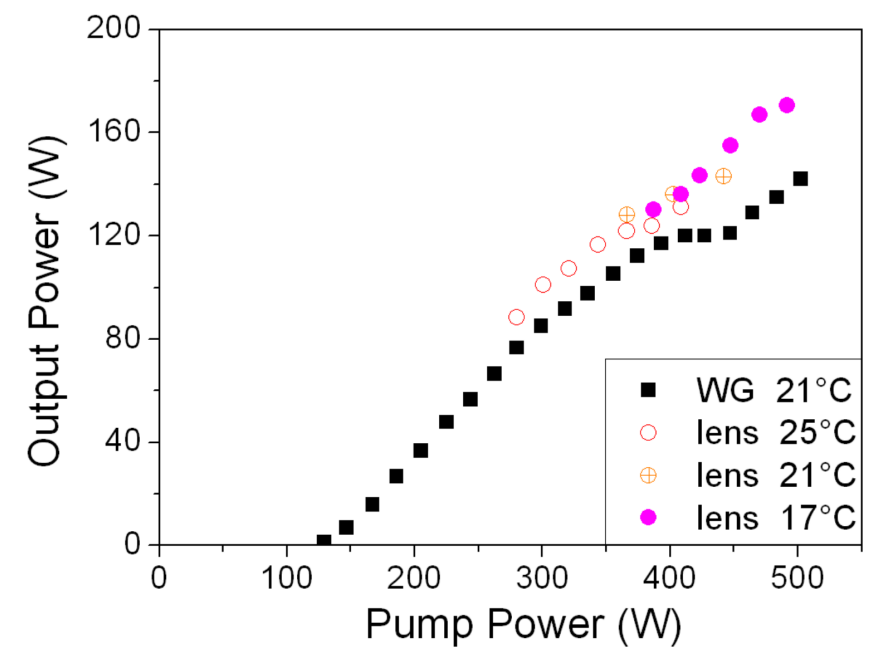

FIG. 3 Output Power versus Pump Power for the edge zig-zagged slab laser with waveguided diode arrays (squares) and lensed diode arrays (circles).

illuminated by two horizontal arrays of four standard $1 \mathrm{~cm}$ wide laser diode bars (either of the JOLD-310-HS-4L or of the JOLD-290-HSC-4L type, produced by Jenoptik Laserdiode $\mathrm{GmbH})$. These laser diode arrays emit at a wavelength between 800 and $810 \mathrm{~nm}$ (with a $2 \mathrm{~mm}$ FBW) depending on the supply current and water cooling temperature. They are passively cooled and are made available either with a cylindrical lens collimation (JOLD-290-HSC-4L) or with a rectangular duct wave-guiding (JOLD- 310-HS-4L).

The active slab is cooled by thermally contacting metallic plates onto the two large area lateral faces, with indium foil interfaces. These slab lateral faces have been optically polished to facilitate the thermal contact and to improve the pump radiation propagation at the same time. The combination of this cooling scheme and of the horizontal diode arrays determines a totally passive cooling of the laser head without any crossing of pump radiation and cooling fluid path. This fact consistently relaxes the requirements on optical and electric properties of the cooling fluid. 
The extraction efficiency properties of this diode pumped slab have been tested in a simple flat- flat cavity determined by a totally reflecting rear mirror and an $80 \%$ output coupler. The optical conversion curves are reported in Fig. 3 for both lens collimated diode arrays and duct wave-guided diode arrays. Measurements were performed only for a limited set of currents and cooling temperatures in the case of lens collimated diodes, in order to avoid an excessive amount of radiation from one array to reach the other, in conditions of non-optimized active medium absorption. A maximum output power of $170 \mathrm{~W}$ was obtained with the lens collimated pump radiation at an optical conversion efficiency equal to $36 \%$. Lens collimated arrays produced a thinner line shaped pumping distribution that better overlaps with the intracavity mode intensity distribution, thus determining a higher efficiency. Each set of experimental data obtained with lenscollimated diodes for different cooling temperatures had an upper limit determined by the extraction curve roll-off. This was correlated with the pump radiation wavelength shift taking place along with the supply current variation, with a consequent absorption reduction. Monitoring the diode spectral emission with a spectrometer (AvaSpec-2048 produced by Avantes BV - NL) we observed the roll- off to happen when reaching a peak emission wavelength around $807 \mathrm{~nm}$ where the Neodimium absorption presents a dip. A longer absorption length obviously changes the thermal gradients distribution. This possibly produces sensible variations in the effective thermal lens and thus affects the optical power conversion efficiency in our zig-zag slab. This effect is visible also in the curve obtained with wave-guided array. Being weaker in this case, it was possible to go past the reduced absorption segment of the curve, by further increasing the current. In the case of lens-collimated pump radiation a sensible reduction in pump power absorption could damage the diodes and this kept us from increasing the current. All these observations bring us to infer that a higher extraction can be obtained from our system reducing the diodes' cooling temperature. In spite of that we decided not to go below $17^{\circ} \mathrm{C}$ to avoid moisture effects, that can also lead to diodes' damage. We thus conclude that we could reach a higher efficiency, using diode arrays with a lower intrinsic emission wavelength.

It is worth noting that, in our case, the optical conversion efficiency is calculated as the ratio between the extracted power and the total diode arrays emitted power. It thus compares quite well with the maximum efficiency reported for the edge-pumped slab in [15] or in the case of end pumped slabs [8]. In fact optical conversion efficiencies are calculated for these latter systems using the pump power at the end of the optical coupling chain. In the case of fibre coupled diode bars, as in [15], a diode-to-fibre optical coupling efficiency of at most $85 \%$ is to be considered to compare with our experiment. Such a correction reduces the optical conversion efficiency $(42 \%)$ to an overall value below the one obtained from our set-up.

To further characterize the potential impact of this laser scheme we measured the propagation quality of the extracted

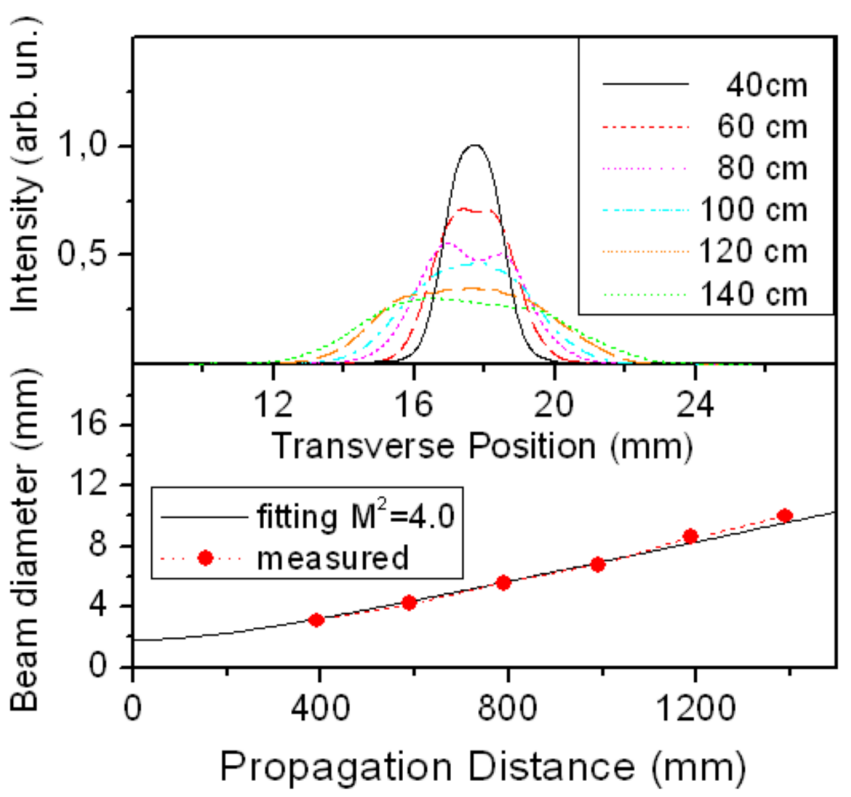

FIG. 4 Beam Profiles in the thin cross-section (upper window) and beam diameters along the propagation path from the output coupler.

beam with particular attention to the transverse direction corresponding to the slab thin cross-section. As already mentioned, the beam quality in the wider cross-section transverse direction is intrinsically worse, given the much larger cross-section dimension, and has to be enhanced with the adoption of a specific cavity scheme such as the Hybrid Stable Unstable Resonator (HSUR) [7]-[9].

For this purpose we acquired beam profiles in the narrow cross-section direction using a linear 256- elements pyroeletric detector array (Spiricon Laserprobe LP-256-11). A sample set of beam profiles acquired at different distances from the output coupler is shown in Fig. 4 along with the calculated beam diameters obtained in the case of a lens-collimated pumping. To evaluate the $\mathrm{M}^{2}$ value of the beam in this transverse direction we refocused the beam with a $600 \mathrm{~mm}$ e.f.l. lens and measured the beam diameters at several propagation distances inside and outside the Rayleigh range of the focused beam.

$\mathrm{M}^{2}$ values have been measured for the different pumping configurations and at different pumping levels. In order to perform measurements at low power levels also in the case of lens-collimated pump radiation we removed one of the two pumping arrays, thus avoiding possible damage due to excessive pump radiation transmitted through the slab, as a consequence of wavelength detuning. The $\mathrm{M}^{2}$ values obtained in our set of experiments are summarized in Fig. 5 . The lenscollimated pump set-up appears to give better results. The more concentrated pump distribution of this set-up produces a smaller transverse section of the gain distribution, and thus a lower number of allowed transverse modes in the resonator, along with a higher thermal lens effect. The former of these to facts seems to prevail in the determination of the beam quality and this could be attributed to the particularly "distributed" 


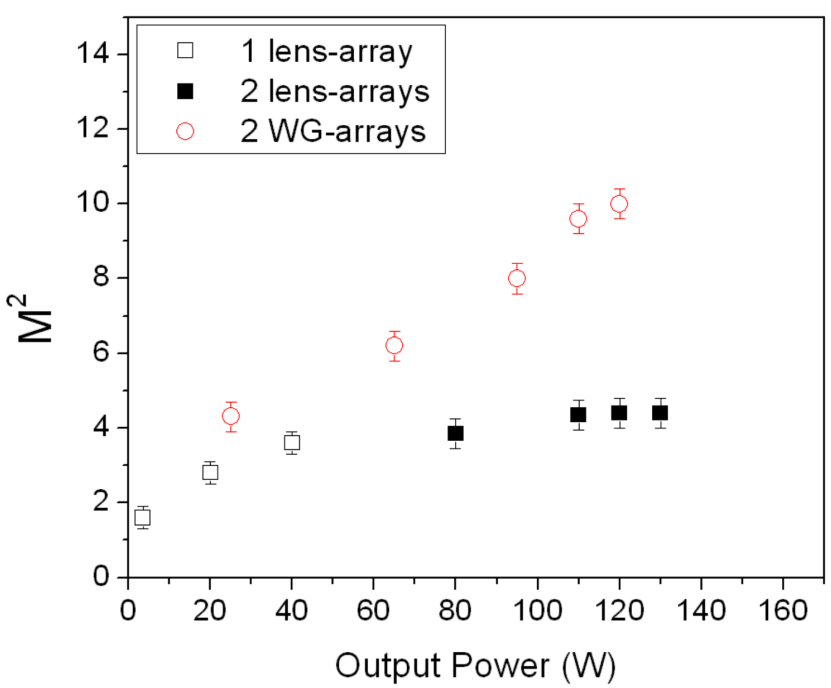

FIG. $5 \mathrm{M}^{2}$ values for the thin cross-sectional direction, obtained in a focused propagation path for different pumping configurations and powers.

feature of the thermal lens in our zig-zag slab scheme.

\section{Conclusions}

These preliminary results allow us to conclude that the "edge zig-zagged" slab configuration is a valuable alternative for high power Diode Pumped Solid State Laser (DPSSL) designs. This design allows the extraction of a reasonably low $\mathrm{M}^{2}$ value beam at a wall plug-efficiency higher than $15 \%$ and at the same time lends itself to the simple realization of pulsed sources from various active media.

This slab scheme presents the advantage of adopting an efficient pumping with extremely simple engineering solutions. Scaling of this design together with the use of ceramic materials could open the range of feasible applications to high energy sources, as for instance those used in Laser Inertial Fusion Energy (LIFE) Programs.

\section{References}

[1] W.S. Martin, and J.P. Chernoch, "Multiple internal reflection face pumped laser", U.S. patent 3,633,126, 1972.

[2] B.J. Comaskey, R. Beach, G. Albrecht, W.J. Benett, B.L.Freitas, C. Petty, D. VanLue, D. Mandinger, and R.W. Solarz, "High Average Power Diode Pumped Slab laser", IEEE J. Quantum Elect. QE-28(4) (1992).

[3] R.J. Shine Jr., A.J. Alfrey, and R.L. Byer, “40-W cW, $\mathrm{TEM}_{00^{-}}$ mode, diode-laser-pumped, Nd:YAG miniature-slab laser", Opt. Lett. 20(5), 459-461 (1995).

[4] D. Mudge, M. Ostermeyer, P. J. Veitch, J. Munch, B. Middlemiss, D. J. Ottaway, and M. W. Hamilton, "Power scalable TEM 00 CW Nd:YAG laser with thermal lens compensation", IEEE J. Sel. Top. Quant. 6, 643-649 (2000).

[5] T. Kawashima et al. , “Diode-Pumped Zig-Zag Slab Laser for Inertial Fusion Energy and Applications", in Advanced Solid-State Photon-
iCS, paper: MB19 (ASSP, USA, 2004).

[6] A. Mandl, and D. E. Klimek, “Textron' s J-HPSSL 100 kW ThinZag Laser Program", in Conference on Laser Electro-Optics: Applications (CLEO_Apps) paper: JThH2 (2010).

[7] K. Kuba, T. Yamamoto, and S. Yagi, "Improvement of slab-laser beam divergence by using an off-axis unstable-stable resonator", Opt. Lett 15(2), 121-123 (1990).

[8] K. Du, N. Wu, J. Xu, J. Giesekus, P. Loosen, and R. Poprawe, "Partially end-pumped Nd:YAG slab laser with a hybrid resonator", 0 pt. Lett. 23, 370-372 (1998).

[9] M. Ciofini, E. Favilla, A. Lapucci, and E. Sani, “Propagation parameters of the beam extracted from a diode pumped Nd:YAG ceramic slab laser with a hybrid stable-unstable resonator", Opt. Laser Technol. 39, 1380-1388 (2007).

[10] A. Lapucci, and M. Ciofini, "Efficiency optimization for a diode pumped Nd:YAG ceramic slab laser", Appl. Optics 44(20), 43884393 (2005).

[11] A. Faulstich, H. J. Baker, and D. R. Hall, "Face pumping of thin, solid-state slab lasers with laser diodes", 0pt. Lett. 21(8), 594-596 (1996).

[12] Y. Liao, K. Du, S. Falter, M. Quade, P. Loosen, and R. Poprawe, "Highly efficient diode-stack, end-pumped Nd:YAG slab laser with simmetrized beam quality", Appl. Optics 36(24), 5872- 5875 (1997).

[13] G.D. Goodno, S. Palese, J. Harkenrider, and H. Injeyan, "Yb:YAG power oscillator with high brightness and linear polarization", 0pt. Lett. 26(21), 1672-1674 (2001).

[14] A.Lapucci, M. Ciofini, E. Favilla, E. Sani, M. De Rosa, G. Mincuzzi, and A. S. D. Rocco, "Quantitative analysis of the thermal distortions in a 100 W CW Nd:YAG ceramic slab laser", J. Eur. Opt. Soc. Rapid 2,07020-1 07020-8 (2007).

[15] T. S. Rutherford, W. M. Tulloch, E. K.Gustafson, and R. L. Byer, "Edge-pumped quasi-three-level slab lasers: design and power scaling", IEEE J. Quantum Elect. QE-36(2), 205-219 (2000).

[16] T. S. Rutherford, W. M. Tulloch, S. Sinha, and R. L. Byer, "Yb:YAG and Nd:YAG edge-pumped slab lasers", Opt. Lett. 26(13), 986-988 (2001).

[17] J. Richards, and A. McInnes, "Versatile, efficient, diode-pumped miniature slab laser", Opt. Lett. 20(4), 371-373 (1995).

[18] A. Mclnnes, and J. Richards, "Thermal effects in a coplanarpumped folded zigzag slab laser", IEEE J. Quantum Elect. QE32(7), 1243-1252 (1996).

[19] J. Lu, K. Ueda, H. Yagi, T. Yanagitani, Y. Akiyama, and A. A. Kamiskii, "Neodymium doped yttrium aluminum garnet $\left(\mathrm{Y}_{3} \mathrm{Al}_{5} \mathrm{O}_{12}\right)$ nanocrystalline ceramics-a new generation of solid state laser and optical materials", J. Alloys Compd. 341, 220-225 (2002).

[20] J. Lu, K. Takaichi, T. Uematsu, J. F. Bisson, Y. Feng, A.Shirakawa, K. Ueda, H. Yagi, T. Yanagitani, and A. A. Kaminskii, "110 W ceramic $\mathrm{Nd}^{3}: \mathrm{Y}_{3} \mathrm{Al}_{5} \mathrm{O}_{12}$ laser", Appl. Phys. B 79, 25- 28 (2004). 